\title{
BMJ Open Why is there variation in the practice of evidence-based medicine in primary care? A qualitative study
}

\author{
Ranita Hisham, Chirk Jenn Ng, Su May Liew, Nurazira Hamzah, Gah Juan Ho
}

To cite: Hisham $\mathrm{R}, \mathrm{Ng} \mathrm{CJ}$, Liew SM, et al. Why is there variation in the practice of evidence-based medicine in primary care? A qualitative study. BMJ Open 2016;6: e010565. doi:10.1136/ bmjopen-2015-010565

- Prepublication history and additional material is available. To view please visit the journal (http://dx.doi.org/ 10.1136/bmjopen-2015010565).

Received 20 November 2015 Revised 5 February 2016 Accepted 8 February 2016

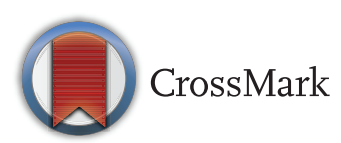

Department of Primary Care Medicine, Faculty of Medicine, University of Malaya, Kuala Lumpur, Malaysia

Correspondence to Ranita Hisham; ranita@um.edu.edu.my

\section{ABSTRACT}

Objective: To explore the factors, including barriers and facilitators, influencing the practice of evidencebased medicine (EBM) across various primary care settings in Malaysia based on the doctors' views and experiences.

Research design: The qualitative study was used to answer the research question. 37 primary care physicians participated in six focus group discussions and six individual in-depth interviews. A semistructured topic guide was used to facilitate both the interviews and focus groups, which were audio recorded, transcribed verbatim, checked and analysed using a thematic approach.

Participants: 37 primary care doctors including medical officers, family medicine specialists, primary care lecturers and general practitioners with different working experiences and in different settings.

Setting: The study was conducted across three primary care settings-an academic primary care practice, private and public health clinics in Klang Valley, Malaysia.

Results: The doctors in this study were aware of the importance of EBM but seldom practised it. Three main factors influenced the implementation of EBM in the doctors' daily practice. First, there was a lack of knowledge and skills in searching for and applying evidence. Second, workplace culture influenced doctors' practice of EBM. Third, some doctors considered EBM as a threat to good clinical practice. They were concerned that rigid application of evidence compromised personalised patient care and felt that EBM did not consider the importance of clinical experience.

Conclusions: Despite being aware of and having a positive attitude towards EBM, doctors in this study seldom practised EBM in their routine clinical practice. Besides commonly cited barriers such as having a heavy workload and lack of training, workplace 'EBM culture' had an important influence on the doctors' behaviour. Strategies targeting barriers at the practice level should be considered when implementing EBM in primary care.

\section{BACKGROUND}

Evidence-based medicine (EBM) has been developed as a strategy to meet this

\section{Strengths and limitations of this study}

- The strength of this study was that it captured a wide range of voices and experiences from primary care doctors across different healthcare settings in Malaysia.

- There is limited research conducted on the implementation of evidence-based medicine in primary healthcare using qualitative methodology.

- The researchers who conducted the interviews were a health sciences librarian and research assistants from a non-medical background, which reduced potential bias in conducting the interviews with the doctors.

- The limitations of this study include doctors being mostly educated and the practices being located in urban centres. These factors may limit the transferability of the findings.

challenge by drawing on scientific evidence to support medical practice. Practising EBM involves making decisions about the care of individual patients by integrating the current best research evidence in a conscientious, explicit and judicious manner; it also takes clinical expertise and patient values into consideration. ${ }^{1}{ }^{2}$ However, although it is more than 20 years since the EBM working group announced a 'new paradigm' for practising clinical medicine, ${ }^{3}$ the use of EBM remains contentious. $^{4}$

Variation in medical practice has become a major topic of enquiry for health service researchers. Healthcare delivery varies among individual clinicians and across geographical areas despite established evidence and clinical practice guidelines. ${ }^{5}$ Practice variations arise from differences in scientific knowledge, doctors' clinical experience and workplace influences including availability of resources, reimbursement systems, practice size and staffing. ${ }^{6}{ }^{7}$ Similarly, the successful implementation of EBM depends on individual doctors' experiences, biases and attitudes alongside professional, organisational and 
workplace factors, all of which can impede the translation of evidence into actual practice. ${ }^{8-12}$

Malaysia has a two-tier healthcare system that consists of a subsidised public sector and a fee-for-service private sector. ${ }^{13}{ }^{14}$ This set-up creates the opportunity to study practice variations across primary care settings. Although EBM has gained widespread acceptance in medicine, ${ }^{15}$ research on this topic in Southeast Asia is scarce. This study aimed to explore the factors, including barriers and facilitators, influencing the practice of EBM across various primary care settings in Malaysia based on the doctors' views and experiences.

\section{METHODS}

\section{Study design}

We conducted a qualitative study to explore the participants' personal views and experiences. We conducted semistructured interviews and focus group discussions with doctors who were grouped according to their clinical setting (public vs private). This approach was taken to ensure homogeneity and to capitalise on shared experiences among the doctors. ${ }^{16}$

\section{Setting}

The interviews were conducted between September 2013 and April 2014 at public health clinics, academic primary care clinics and private general practice clinics in Klang Valley, Malaysia. In Malaysia, the public sector consists of government-subsidised hospitals and health clinics, while the private sector comprises fee-for-service hospitals and clinics. In this study, doctors were purposively sampled from both settings to achieve maximum variation based on EBM knowledge, working experiences and settings.

\section{Participants, recruitment and sampling}

Doctors working in public and private healthcare clinics were recruited through email and telephone invitations. Researchers arranged to interview the doctors at a time and location of their convenience. Most of the interviews were held at the doctors' workplace except for one group of general practitioners which was interviewed after a clinical training workshop. The participants included medical officers (doctors who work in the public sector and do not have a postgraduate qualification), family medicine specialists, primary care lecturers and general practitioners working in primary care settings. Recruitment was continued until data saturation was achieved. Trained qualitative researchers conducted focus group discussions and individual in-depth interviews using a semistructured topic guide. All participants received and went through the participant information sheet before giving written consent.

\section{Data collection}

An interview topic guide (see online supplementary file 1) was developed and subsequently modified in response to settings and themes that emerged during the interviews. Open-ended questions were asked during the interview to explore views and understanding towards EBM (attitudes), how their practice is being influenced by their peers (subjective norms) and their workplace culture (control). The same topic guide was used and the same information about the study was given for both interviews and focus groups. The interview questions were developed from findings from previous studies and expert opinions and based on the Theory of Planned Behaviour (TPB). TPB is an extensively used psychological model for understanding human behaviour. ${ }^{17}$ It suggests that people are far more likely to behave in a specific way if they form a conscious intention to do so and this intention is the major determinant of whether the behaviour will happen. Intention is influenced by attitude, subjective norms and selfefficacy. Each interview lasted 45-60 min. All identifiers were removed and data were kept confidential.

\section{Data analysis}

A thematic analysis approach was used for data analysis. We used in-depth interviews, focus group discussions and field notes to triangulate the data. All interviews were audio recorded, transcribed verbatim and checked. The transcripts, supplemented by reflective field notes, were read and reread for familiarisation and immersion. Three researchers (RH, NH and GJH) independently coded two interviews line by line to develop an initial list of codes. Subsequent interviews were coded using this list, and new themes which emerged from these interviews were added to the list on consultation with the research team. Nodes with similar themes were grouped to form categories. The coding framework was checked independently by SML and CJN, and discrepancies were resolved via discussion and consensus. Themes were then refined by removing or recoding redundant and infrequent nodes to produce the final themes. Data saturation was obtained when new codes ceased to emerge from the data and repeated coding occurred with the same codes. Data analysis was facilitated by the use of the computer-assisted qualitative analysis software Nvivo V.10 to help sort, arrange and classify the data.

\section{RESULTS}

There were 37 primary care physicians who participated in six focus group discussions $(\mathrm{n}=31)$ and six individual in-depth interviews. The demographic and clinical backgrounds of the participants are presented in table 1 . We identified three core themes that influenced the implementation of EBM in practice: (1) lack of knowledge and skills in searching and applying evidence, (2) EBM culture in a doctor's workplace, and (3) the perception of EBM as a threat to good clinical practice. The summary of coding framework is presented in table 2. 
Table 1 Demographic and clinical backgrounds of the participants

\begin{tabular}{ll}
\hline Characteristics & $\begin{array}{l}\text { Number of } \\
\text { participants }(\mathbf{n}=\mathbf{3 7})\end{array}$ \\
\hline Age (years) & $26-51$ \\
Gender & \\
$\quad$ Male & 11 \\
$\quad$ Female & 26 \\
Position & 7 \\
$\quad$ Family medicine specialists & 22 \\
$\quad$ Medical officers & 8 \\
$\quad$ General practitioners & \\
Workplace & 15 \\
$\quad$ Academic primary care settings & 14 \\
Public healthcare & 8 \\
$\quad$ Private health clinics & $1-28$ \\
Years of practice (years) & \\
Ever attended an EBM course & 12 \\
$\quad$ Yes & 25 \\
$\quad$ No & \\
\hline EBM, evidence-based medicine. &
\end{tabular}

\section{Lack of knowledge and skills in searching and applying evidence}

First, there was a lack of knowledge and skills in searching for evidence and applying it to patients. Most of the doctors found statistics difficult, and they were only interested in the final conclusion of research papers.

We like to do it $[\mathrm{EBM}]$, the problem is when it comes into statistics we get scared, I'll get stuck and can't proceed any longer. (P22, female, 35 years old, medical officer, academic primary care setting)

Table 2 Core themes that influenced the implementation of EBM practice

\begin{tabular}{|c|c|}
\hline Themes & \\
\hline $\begin{array}{l}\text { (1) Lack of knowledg } \\
\text { applying evidence } \\
\text { (2) EBM culture in th }\end{array}$ & $\begin{array}{l}\text { and skills in searching for and } \\
\text { workplace }\end{array}$ \\
\hline $\begin{array}{l}\text { Academic primary } \\
\text { care settings }\end{array}$ & $\begin{array}{l}\text { Doctors' attitude towards EBM } \\
\text { Access to EBM resources, } \\
\text { training and expertise }\end{array}$ \\
\hline $\begin{array}{l}\text { Private health } \\
\text { clinics }\end{array}$ & $\begin{array}{l}\text { Patients' preference } \\
\text { Doctors prefer to treat patients } \\
\text { based on experience } \\
\text { - Lack of resources }\end{array}$ \\
\hline $\begin{array}{l}\text { Public health } \\
\text { clinics }\end{array}$ & $\begin{array}{l}\text { Doctors' adherence to guidelines } \\
\text { and specialist's opinion } \\
\text { - Lack of time } \\
\text { - Drugs availability } \\
\text { - Patients' compliance } \\
\text { - Access to information } \\
\text { - Outdated guidelines }\end{array}$ \\
\hline
\end{tabular}

EBM, evidence-based medicine.
I didn't understand what are odds ratio, relative risk, and all that. This is like a barrier for us to appraise a paper. I was very naïve but now I understand. So, I think knowledge is very important especially knowledge in appraising a journal paper (P8, female, 33 years old, medical officer, academic primary care setting).

Those statistics it's my weakest! I'll just leave it if I don't know and because clinically we don't use it, to be frank with you. Basically I think this $[\mathrm{EBM}]$ are mainly for those who are interested in finding out the answer. I think I just want to know the p-value, what the conclusion is and what we need to do. (P14, male, 32 years old, medical officer, public health clinic)

I have to admit that it is hard to understand the methods and statistics. Not everything I will understand, if I don't understand I will attend the workshops or talks, and then I will probably understand. Personally I hate to read! I rather listen than to read. (P37, female, 48 years old, senior private general practitioners)

In addition, some doctors found it very difficult to look for evidence from credible sources. This was attributed to a lack of searching skills.

"We probably wouldn't be as good as librarians to search for information. So how we coped is that we look for information the way we looked for it, so whether it's adequate or inadequate, we just have to do it. Because you know I think the problem is that we could never get very good at one skill because there's too many things that we have to do." (P36, female, 45 years old, lecturer, academic primary care setting)

\section{EBM culture in the workplace}

Workplace culture influenced doctors' practice of EBM. In this study, work environment either facilitated implementation of EBM or stood as a barrier to it.

\section{Academic primary care setting} Doctor's attitude towards EBM

Most of the doctors from the academic primary care setting tended to have better knowledge and more positive attitudes towards EBM because they were expected to practise EBM. Protected time was given for research, which encouraged the use of evidence.

I think the good thing about our work place is actually we've been scheduled, meaning that one day we'll have clinic's day and then maybe certain days we do research followed by teaching on the other days, so I think yes we are coping. (P36, female, 45 years old, academic primary care setting)

Most of the primary care specialists were ready to answer clinical questions and support their trainees.

Yes we can help them [trainees], by hook or by crook isn't it? It's basic. I think we also gone through the same thing when we were in training, so of course we try to 
help them the best that we can. But there are certain things that we don't know, so we ask other colleagues as well but sometimes if I really don't know I will just tell the MO [medical officer] to give me a few minutes to look up for the answer. I sometimes go to the internet and reliable websites so that I can sort of give a guide to that MO regarding the query. (P30, female, 40 years old, academic primary care setting)

\section{Access to EBM resources, training and expertise}

The availability of an online library was helpful for retrieving full-text journals. However, some of the doctors were unsure how to search, appraise and apply the evidence in their routine clinical practice.

Because it needs lots of references, it's not that one topic, we need to cross reference and then finally come to a conclusion, and it's not easy with the patient in the room. (P5, female, 33 years old, academic primary care setting)

Support from top management was necessary for EBM implementation.

An important thing about implementing EBM is I think some of it is actually from top down isn't it? There must be some kind of structures that we could adapt this quickly? Like J said a new medicine or the new way of doing things are either not available, too costly or nobody supporting. The support network has to be there either from the administrators or the people around you. Everybody should be supportive if you are going to implement EBM. (P36, female, 45 years old, academic primary care setting).

\section{Private health clinics}

Patient's preference

A few doctors felt frustrated because patients wanted them to prescribe medications which were not recommended in the guidelines. Patients would otherwise get their own medication from the pharmacies. Patients got medical information from unreliable sources and assumed that expensive drugs are better.

I try my very best to practice evidence-based. If the patient is against it and still insists, I will give them the antibiotics or I will say maybe you don't have to take the antibiotics today and tomorrow. You take it the third day or something like that. (P24, female, 43 years old, private general practitioner).

They self-treat a lot. Change a lot of their own medication, and come up with articles from websites. (P28, female, 43 years old, private general practitioner).

Because they get it [medicine] easily outside, they ask about it here [clinic] and get it outside [pharmacy]. When we say we cannot give because it is not appropriate and not according to guideline and it is not recommended. They will be like 'why, why, why?' They keep asking you and you keep explaining to them and sometimes we don't know whether to just listen to the patient's concern and give or still practice the evidencebased. (P4, female, 33 years old, medical officer, academic primary care setting).

\section{Doctors prefer to treat patients based on experience}

Some general practitioners treated their patients based on experience. According to them, every doctor has the right to treat patients based on what they think is best for them. They were also 'comfortable' with what they had been practising and did not see a reason to change their practice.

Well, when it's in the guidelines, I can utilize it. But I don't, I'm not an avid reader. Whatever has enough grounds and I can apply it to my practice, yes. Not to say cannot. I'm comfortable with certain things that I'm already doing. You know it works for the patients and of course it does. What the patient are expecting from meeting you. So, it's more based on that other than EBM itself. (P17, female, 28 years old, private general practitioner)

When I haven't done the FM [Masters in Family Medicine], I wouldn't be exposed to EBM, I will just be in my comfort zone. Doing whatever I've known for quite some time. So those days we are just using our own EBM! [Laugh.] (P20, female, 43 years old, private general practitioner)

\section{Lack of resources}

There are many new methods of managing patients; therefore, doctors need more financial support from their administration for them to practise. There are limited drugs in general practitioner clinics and healthcare clinics due to insufficient budgets; more advanced drugs are only available in hospitals. Patients cannot afford to buy expensive drugs, so they will get different drugs from hospitals which might not be evidencebased. Private general practitioners felt that there is lack of support in private clinics, especially in relation to drug availability, with the result that the standard of emergency care in private clinics has to be very basic management.

Actually we don't have many drugs here; we have the basic ones only. So, anything advance we'll refer to the hospital. (P29, male, 46 years old, private senior general practitioner)

\section{Public health clinics}

\section{Doctors' adherence to guidelines and specialist's opinion}

Most of the doctors in the public setting seemed to be more dependent on guidelines and specialists. They were less likely to search for clinical evidence themselves.

Whatever that is already in the CPG [Clinical Practice Guidelines] guideline or any guideline, it's already evidence-based. Follow it and you'll be fine. (P9, female, 33 years old, medical officer) 
I think I will just follow what all of them [Family Medicine Specialist] said. So, the first and fast available reference would be your FMS if she is around. (P13, male, 27 years old, medical officer)

Most of the family medicine specialists were receptive towards EBM and were willing to search for evidence themselves.

I would say yes of course EBM is helpful and useful. $\mathrm{Hmm}$, but sometimes you have to take it with a pinch of salt. You can't just totally swallow and apply the evidence because different situation you have to tailor different treatment, you have to tailor the local situation. (P19, female, 47 years old, family medicine specialist)

Yes, if I am stuck, I have to look for the information even though the patient is in front of me because I don't want to make mistake! (P2, female, 43 years old, Family Medicine Specialist)

\section{Lack of time}

Most of the doctors found it hard to search for answers; they felt that the biggest challenge to the practice of EBM was 'lack of time'. It took time to appraise a paper, which required them to read and digest it carefully.

Oh! We send out the patient and take that time to quickly look up the internet in between or what's the evidence for this and when the patient returns, I can tell oh yes this is the evidence. As fast as possible in that short period of time! (P11, female, 31 years old, medical officer)

Most of the doctors felt that their workload was too heavy, and they could not cope with implementing EBM into their practice. There were too many patients, and the workload was high. Because our situation with number of patients and the workload ... whether we can implement it [EBM], that's a different story, it's whether we can use it at that point of time. (P6, female, 47 years old, medical officer)

\section{Drugs availability}

Doctors in these settings also faced the same problem of drug restrictions as the private general practitioner clinics.

Drug restrictions and availability of the medication is a problem here. Most of them are available in the hospital. (P13E, female, 34 years old, medical officer)

I worked in the Klinik Kesihatan [public health care] before this, sore throat medicine not available, most of the medicines is not available, medicine which makes you sleepy only available. No evidence-based at all. (P3, female, 43 years old, family medicine specialist)

\section{Patients' compliance}

In addition, patients tended to self-prescribe. They change their medication and refused the medication prescribed by doctors. This circumstance definitely influenced doctors' practice.

We are in a population where they are all retired professionals. They Google a lot and they seek opinion from people of different backgrounds and they formulate opinion themselves. (P9, female, 33 years old, medical officer)

I do get a bit defensive and intimidating when patient change their medication and sometimes they don't know the name of the medication. They refuse to take some of the medication I prescribe, if they refuse, I would refer them to government hospitals, and then yes it would definitely change my management a lot. (P7, female, 27 years old, medical officer)

\section{Access to information}

In public health clinics, journal papers are mostly accessible through some hospital libraries and annual subscriptions given by drug companies. Some of the doctors therefore relied on journals which were free or published in non-subscription journals, or they would just read the abstracts.

The availability of the journal is like how accessible is it, certain things must be paid for, whether you have access to them or not and that's based on individual ... either you want to refer to textbooks or journals. (P12, female, 42 years old, family medicine specialist)

\section{Outdated guidelines}

Some of the doctors were also frustrated with the guidelines because of the limited copies available in their settings. Some of the Malaysian CPGs were outdated, while others were not user friendly.

Our CPGs being so outdated and we are still being told to use the CPGs, so that is the primary problem. Then, the availability of medications in our clinics such as diabetes. It's so limited, they are basing it on the CPG, but the world is 50 years ahead of us. We are still using longacting sulphonylureas which are evidently bad for health. Yes we are still using it. It's like $100 \%$ used here. (P, female, 36 years old, family medicine specialist)

For a simple CPG like a MOH's (Ministry of Health) CPG in sore throat back dated to 2003 I think. And it's very simple like hardly anything in there, and you have to follow, so I can't find what I'm looking for. I mean, how do I treat? They don't give us a proper way to follow; it's not available in the CPG. (P16, female, 28 years old, medical officer)

\section{EBM as a threat to good clinical practice}

Some doctors, particularly those from the private practice, considered EBM a threat to good clinical practice. They were concerned that rigid application of evidence compromised individualised patient care. They felt that EBM did not incorporate doctors' clinical experience. 
One of the senior general practitioners felt that EBM restricted his practice as a doctor. He perceived EBM as adhering to the guidelines and research-based answers.

But I feel that [EBM] limits your practice also. Limits like you got no choice. So, I feel that we are just totally guideline based; the role of the doctor is diminished. There are no more roles for me in the medical care anymore. I am superfluous. Someone comes in with diabetes, you have to give metformin. That's what the guideline said. Where is your role as a doctor? I should be a clerk! (P29, male, 46 years old, senior private general practitioner)

Most of the senior general practitioners treat patients based on experience. According to them, every doctor has the right to treat patients based on what he or she thinks is the best for the patient, which is based on experience.

Why practice EBM? I practice EBM with a touch of reality. [Laugh] No, I don't totally practice it. I want to be aware of what is EBM, and as I get older, I think experience becomes a bigger factor. As a younger doctor, I followed EBM more; I think as you get older you tend to deviate a bit in your practice. So, that has been what I felt, how I've changed. I mean I used to be "Oh! Evidence-based, evidence-based and evidence-based!". (P29, male, 46 years old, senior private general practitioner)

\section{DISCUSSION}

A key finding from this study is that practising EBM in primary care is still challenging in Malaysia. From our analysis, the practice of EBM is influenced by three key elements: lack of knowledge and skills in searching for and applying evidence, EBM culture in the workplace and EBM as a threat to a good clinical practice. The novelty of this study is that various primary healthcare settings were included and each setting had its own issues.

Doctors have different views about how best to practise medicine. Senior doctors seemed to focus more on experience, which they considered better than practising EBM because their knowledge is the basis of their skills. Doctors in public and private health clinics indicated that they lacked knowledge and skills on how to practise EBM, and some of them had not been exposed to EBM. Most of these doctors relied on guidelines and referred to colleagues and specialists rather than searching for evidence themselves. Some of them were unsure about the concept of EBM, had difficulties searching for evidence, doubted the appraisal process and found it hard to understand statistics in research papers. A systematic review by Swennen $e t a l^{18}$ highlighted that lack of knowledge and skills strongly hindered the practice of EBM in four areas: asking answerable questions, searching best evidence, appraising evidence and translating evidence. Accessibility to evidence is another issue, which is a barrier to doctors searching for evidence. Doctors in the academic primary care settings found it easy to retrieve full-text papers compared to doctors in the private primary care setting.

Work setting was also a factor that influenced the practice of EBM. Even in settings where EBM is well supported, doctors still do not practise it, especially when there is a heavy workload. Doctors may do it because they feel that they are expected to, but they may not understand the actual meaning of EBM. In our study, doctors in the academic primary care setting had greater exposure to EBM; their workplace EBM culture was supported by the organisation. The non-academic settings, however, had a different work culture which often hindered rather than facilitated doctors' practice of EBM. Unfortunately, there is often minimal support from the organisation and it depends on individual doctors to decide whether or not they would like to practise EBM. This decision is influenced by their workload and time constraints. In this study, support from higher authorities seemed to be lacking. The responsibility for implementing EBM in the workplace should not fall solely on the individual healthcare practitioner. Healthcare organisations need to provide an environment conducive to implementing EBM in order for their staff members to effectively provide the highest level of patient care. ${ }^{19-21}$ Organisations should also be wary of creating a 'blame culture' whereby healthcare personnel who attempt to work flexibly within protocols are reprimanded; this response may prevent the development of new approaches, such as the implementation of EBM, in existing practices. ${ }^{22} 23$

Patients are becoming more knowledgeable as health information is now easily accessible through the internet. ${ }^{24}$ Doctors in private settings highlighted this issue the most because patients are paying for their medication and they demand the best treatment. The majority of the doctors stated that patients demand certain medication, which indirectly allows patients to influence the doctors' decision-making, which in turn affects the practice of EBM. ${ }^{725}$

Patient values may conflict with doctor's advice based on evidence. This may be overcome by using the shared decision-making model where both parties discuss, negotiate and agree on the decision. ${ }^{26}$ Private healthcare settings have been struggling to fulfil the need to practise EBM due to financial constraints; problems remain with lack of drug resources. They cannot follow the recommended treatment or drugs based on evidence if the setting cannot provide the resources. Most of the evidence is based on different population, and it is too expensive. A limited choice of drugs available also affects, public healthcare settings and the limitation restricts doctors' implementation EBM with their patients. For example, the doctors are only allowed to use what is allocated; they can give an ACE inhibitor to a patient with diabetes with proteinuria, but if the patient cannot tolerate it, angiotensin receptor blockers (losar$\tan )$ is given. The doctors can only prescribe losartan 
for 100 patients because of a limited patients' quota. For that reason, doctors face barriers to practice based on evidence.

The practice of EBM also depends on an individual's attitude. In this study, doctors' personal attitude influenced their practice of EBM. Some doctors considered EBM as a threat to good patient care because they felt that drawing on their experience was a more effective way to treat patients. In these doctors' view, EBM is based too rigidly on research and authority and does not take patient's individual characteristics into consideration. It restricts clinical practice and limits the choices. This negative perception increases doctors' resistance to accepting EBM. ${ }^{27}$ Bandura $^{28}$ suggests that personal factors, environmental factors and behaviour simultaneously influence each other, which may strongly influence behaviour. Behaviour could be improved by removing the barriers created by gaps and inadequacies in these factors. However, attitude, if negative, can have a devastating influence on behaviour. ${ }^{29} \mathrm{~A}$ negative attitude of staff towards EBM, an 'expert-based' and nonreciprocal communication, prevents both the exchange of information and discussion about medical decisions. Respectful and reciprocal communication among doctors was reported to be a strong facilitator of EBM. Such a culture helped doctors to discuss and challenge medical decisions across career stages, medical disciplines and settings. ${ }^{18}$

\section{Strengths and limitations}

The strength of this study was that it captured a wide range of voices and experiences from primary care doctors across different healthcare settings in Malaysia. There is limited research conducted on the implementation of EBM in primary healthcare using qualitative methodology. In addition, the researchers who conducted the interviews were a health sciences librarian (RAH) and research assistants (HGJ and NAH) from a non-medical background with a Master's qualification, which reduced potential bias in conducting interviews with doctors. It was also reviewed by two academicians who are experienced researchers (NCJ and LSM) with a PhD qualification. We believe that findings from this qualitative study will help inform the development of interventions to overcome barriers to the practice of EBM in primary care. The limitations of this study include doctors being mostly educated and the practices being located in urban centres. These factors may limit the transferability of the findings.

\section{Implications for practice and research}

Despite some of the doctors having positive attitudes about EBM and being aware of its importance, most primary care doctors do not regularly practice EBM because of barriers such as a heavy workload and a lack of training and support from the organisation. In addition, the practice of EBM depends on the healthcare setting and workplace culture. The practice of EBM may be facilitated by providing more support to doctors in terms of training, time, incentives and readily accessible evidence. Therefore, incorporating EBM into routine practice requires a change in the workplace culture and support system. Clinicians should work with fellow colleagues, clinic administrators and health authorities to find ways to facilitate the implementation of EBM. To reduce variations in practising EBM, a healthcare organisation should commit to provide training, access to EBM resources and support services for the primary care doctors.

\section{CONCLUSION}

Despite some of the doctors having positive attitudes about EBM and being aware of its importance, most primary care doctors do not regularly practise EBM because of barriers such as a heavy workload and a lack of training and support from the organisation. In addition, the practice of EBM depends on the healthcare setting and workplace culture. The practice of EBM may be facilitated by providing more support to doctors in terms of training, time, incentives and readily accessible evidence.

Acknowledgements The authors thank the healthcare professionals who participated in this study and the University of Malaya for funding this project.

Contributors RH wrote the first draft of the paper and led the revision of the paper, facilitation of data collection interviews and focus groups, transcription and data analysis. CJN and SML were involved in the conception, study design, development of the interview topic guides used for data collection, analysis of the data for reporting, interpretation of the data, and review of the paper. GJH and NH contributed to the facilitation of the data collection interviews and focus groups, transcription and data analysis. All authors have read and approved the final manuscript.

Funding This study was supported by a High Impact Research (HIR) Grant, University of Malaya with grant number (E000078).

Competing interests None declared.

Ethics approval This study received ethics approval from the University of Malaya Medical Centre Medical Ethics Committee (MREC: 962.9).

Provenance and peer review Not commissioned; externally peer reviewed.

Data sharing statement No additional data are available.

Open Access This is an Open Access article distributed in accordance with the Creative Commons Attribution Non Commercial (CC BY-NC 4.0) license, which permits others to distribute, remix, adapt, build upon this work noncommercially, and license their derivative works on different terms, provided the original work is properly cited and the use is non-commercial. See: http:// creativecommons.org/licenses/by-nc/4.0/

\section{REFERENCES}

1. Guyatt G, Rennie D. Users' guide to the medical literature: essentials of evidence-based clinical practice. IL: AMA Press, 2002.

2. Straus SE, Richardson WS, Glasziou P, et al. Evidence-based medicine: how to practice and teach EBM. 3rd edn. Edinburgh: Elsevier Science, 2005.

3. Evidence-Based Medicine Working Group. Evidence-based medicine. A new approach to teaching the practice of medicine. JAMA 1992;268:2420-5.

4. Greenhalgh T, Howick J, Maskrey N. Evidence based medicine: a movement in crisis? BMJ 2014;348:g3725.

5. Love T. Variation in medical practice: literature review and discussion. Wellington: Sapere Research Group, 2013. 
6. Hjerpe P. Investigating practice variation in a changing primary care: a multilevel perspective on The Skaraborg Primary Care Database [Doctoral Dissertation Series]. Sweden: Lund University, 2011.

7. Molstad S, Lundborg CS, Karlsson AK, et al. Antibiotic prescription rates vary markedly between 13 European countries. Scand J Infect Dis 2002;34:366-71.

8. Brady N, Lewin L. Evidence-based practice in nursing: bridging the gap between research and practice. J Pediatr Health Care 2007;21:53-6.

9. Fineout-Overholt E, Melnyk BM, Schultz A. Transforming health care from the inside out: advancing evidence-based practice in the $21 \mathrm{st}$ century. J Prof Nurs 2005;21:335-44.

10. Funk SG, Champagne MT, Wiese RA, et al. Barriers: the barriers to research utilization scale. Appl Nurs Res 1991;4:39-45.

11. Newman M, Papadopolous I, Sigsworth J. Barriers to evidencebased practice. Intensive Crit Care Nurs 1998;14:231-8.

12. Retsas $\mathrm{A}$. Barriers to using research evidence in nursing practice. $J$ Adv Nurs 2000;31:599-606.

13. Chan GC, Teng CL. Primary care doctors' perceptions towards evidence-based medicine in Melaka State: a questionnaire study. Med J Malaysia 2005;60:130-3.

14. Council MHT. Healthcare Industry in Malaysia Malaysia: Ministry of Health Malaysia, 2013. http://www.mhtc.org.my/en/healthcareindustry-in-malaysia.aspx.

15. Ghahremanfard F, Nassaji M, Mirmohammadkhani M, et al. Knowledge and attitude toward evidence-based medicine among medical students in Semnan, Iran. J Evid Based Med 2014;7: 32-7.

16. Kitzinger J. Qualitative research: introducing focus groups. BMJ 1995;311:299-302.

17. Ajzen I. The theory of planned behavior. Organ Behav Hum Decis Process 1991;50:179-211.
18. Swennen MH, van der Heijden GJ, Boeije HR, et al. Doctors' perceptions and use of evidence-based medicine: a systematic review and thematic synthesis of qualitative studies. Acad Med 2013;88:1384-96.

19. Cummings GG, Estabrooks CA, Midodzi WK, et al. Influence of organisational characteristics and context on research utilization. Nurs Res 2007;56:S24-39.

20. Marchionni C, Ritchie J. Organizational factors that support the implementation of a nursing best practice guideline. J Nurs Manag 2008;16:266-74.

21. Wallin L, Ewald U, Wikblad K, et al. Understanding work contextual factors: a short-cut to evidence-based practice? Worldviews Evid Based Nurs Res 2006;3:153-64.

22. Gray M Joy E, Plath D, et al. Implementing evidence-based practice: a review of the empirical research literature. Res Soc Work Pract 2013;23:157-66.

23. Scott SD, Pollock $C$. The role of nursing unit culture in shaping research utilization behaviors. Res Nurs Health 2008;31:298-309.

24. Laugesen J, Hassanein K, Yuan Y. the impact of internet health information on patient compliance: a research model and an empirical study. J Med Internet Res 2015;17:e143.

25. Branthwaite AJCP. Pan-European survey of patients' attitudes to antibiotics and antibiotic use. J Int Med Res 1996;24:229-38.

26. Charles C, Gafni A, Whelan T. Shared decision-making in the medical encounter: what does it mean? (or it takes at least two to tango). Soc Sci Med 1997;44:681-92.

27. Parker M. False dichotomies: EBM, clinical freedom, and the art of medicine. Med Humanit 2005;31:23-30.

28. Bandura A. Social foundations of thought and action: a social cognitive theory. Englewood Cliffs, NJ: Prentice-Hall, 1986.

29. McColl A, Smith H, White P, et al. General practitioner's perceptions of the route to evidence based medicine: a questionnaire survey. BMJ 1998;316:361-5. 